Article

\title{
Experimental Identification and Vibration Control of A Non-Collocated Piezoelectric Flexible Manipulator Using Optimal Multi-Poles Placement Control
}

\author{
Junqiang Lou 1,*, Jiangjiang Liao ${ }^{1}$, Yiling Yang ${ }^{2}$, Yanding Wei ${ }^{2}$ and Guoping Li ${ }^{1}$ \\ 1 College of Mechanical Engineering and Mechanics, Ningbo University, Ningbo 315211, China; \\ neverstop2008@126.com (J.L.); liguoping@nbu.edu.cn (G.L.) \\ 2 China Key Laboratory of Advanced Manufacturing Technology of Zhejiang Province, \\ School of Mechanical Engineering, Zhejiang University, Hangzhou 310058, China; \\ yangyiling@nbu.edu.cn (Y.Y.); weiyd@zju.edu.cn (Y.W.) \\ * Correspondence: loujunqiang@nbu.edu.cn; Tel.: +86-15958885449
}

\begin{abstract}
This paper presents experimental identification and vibration suppression of a flexible manipulator with non-collocated piezoelectric actuators and strain sensors using optimal multipoles placement control. To precisely identify the system model, a reduced order transfer function with relocated zeros is proposed, and a first-order inertia element is added to the model to compensate the non-collocation. Comparisons show the identified model match closely with the experimental results both in the time and frequency domains, and a fit of $97.2 \%$ is achieved. Based on the identified model, a full-state multi-poles placement controller is designed, and the optimal locations of the closed loop poles are determined. The feasibility of the proposed controller is validated by simulations. Moreover, the controller is tested for different locations of the closed loop poles, and an excellent performance of the optimal locations of the closed loop poles is shown. Finally, the effectiveness of the proposed controller is demonstrated by experiments. Results show that the vibrations of the expected modes are significantly diminished. Besides, vibrations of the higher modes are also slightly suppressed. Accordingly, multi-mode vibrations of the manipulator are well attenuated, and the tip displacement converges quickly with the proposed method.
\end{abstract}

Keywords: experimental identification; multi-poles placement control; smart flexible manipulator; active vibration control; non-collocation

\section{Introduction}

In the fields of aerospace, robotics, civil engineering and other industries, flexible structures are prevailing due to their advantages of lightweight and lower energy consumption. However, flexible structures are highly resonant systems and exhibit the inherent property of vibration in presence of external disturbances, which leads to a deterioration of positioning accuracy and efficiency. Thus, vibration suppression of those multi-mode structures becomes imperative [1]. In recent years, smart materials have been extensively used for vibration control of flexible structures. Those materials include shape memory alloys, piezoelectric transducers and so on [2]. Among them, piezoelectric materials have the advantages of small volume, fast response and can be easily integrated onto the host structures. As a result, active vibration control of smart structures utilizing piezoelectric materials has become more attractive [3].

To obtain a better performance for vibration suppression of flexible structures, both accurate dynamic modeling and suitable controller design are required. And controller design often relies on accurate modeling of the system dynamics [4]. In most previous studies, various efficient approaches have been proposed to establish the mathematical model of smart flexible structures. In general, dynamic behaviors of flexible structures can be obtained by analytical modeling based on partial differential equations (PDEs) [5]. But the analytical model is less effective for the requirement of high- 
precision, and the introduction of piezoelectric (PZT) actuators/sensors adds complexity to the analytical modeling. In some cases, it is intractable, even impossible to find the system differential equations. Numerical approaches based on finite element method (FEM) are developed to obtain equivalent finite-dimensional models of smart structures, and yield fairly accurate results [6]. However, these approaches always require intensive mathematical derivations, which are usually labor intensive and time consuming. Meanwhile, these modeling techniques are sometimes hindered by factors such as the assumption of ideal boundary conditions, perfect bonding between the host and actuators/sensors, and so on [7].

An alternative modeling approach is system identification, which captures the input-output dynamics of smart structures using experimental data. Moreover, the issues of the sensor/actuator dynamics, signal conditioners and control unit are all included in the experimental model. Thus, the established model faithfully represents the dynamics of the flexible structures incorporated with smart materials, and the tangled mathematic calculations are also avoided. The identification process can be conducted in the frequency domain, or in the time domain. Among those time domain methods, Bu et al. [8] presented an experimental model of a flexible beam system incorporated with piezoceramic actuators based on the ARMAX (auto-regressive moving average exogenous) model. Abreu et al. [9] derived a time-domain state space model of a flexible structure using the observer/kalman filter identification method together with an eigensystem realization algorithm. Tavakolpour et al. [10] employed the genetic algorithm (GA) to identify the model parameters of a linear difference equation for a rectangular flexible plate system. Afshari et al. [11] utilized a prediction error method to obtain the ARMAX model of a flexible beam bonded with piezoceramic actuator/sensor, and implemented a model reduction method to find the amenable reduced order. Pradhan and ubudhi [12] developed a nonlinear ARMAX model of a planar two-link flexible manipulator system, and designed a nonlinear adaptive controller based on the identified model. As flexible structures are distributed parameter systems, thus have multiple vibration modes, which display highly resonant behavior near to the structures' natural frequencies. For a vibration mode, the frequency and damping of the underlying sinusoidal behavior are the basic parameters to be estimated. San-Millan and Feliu [13] presented an algebraic derivative approach for online identification of the resonant frequencies of a flexible beam in the presence of noise and an offset. Qiu et al. [14] conducted an acceleration sensor based identification of modal frequencies for plate structures, including the first two bending and torsional modes. In order to estimate the model parameters of a flexible structure with collocated sensors/actuators, Bhikkaji et al. [15] proposed a novel frequency domain subspace identification scheme using the negative imaginary approach. To obtain the experimental model of a flexible plate, several frequency domain subspace identification algorithms with the instrumental variable idea were addressed by Ahmadizadeh et al. [16]. In addition, Orszulik and shan [17] studied the application of the GA to identify the transfer function of a flexible manipulator system with collocated piezoelectric sensor/actuator.

Due to the inherent distributed nature, flexible structures have an infinite number of modes. But it is impossible to model and active control all these modes due to the computational and control limitations. In practice, the reduced order models of flexible structures are always developed by modal truncation, where only the dominant modes are considered [18]. Consequently, the problem of spillover associated with the un-modeled but often non-negligible modes is unavoidable, and the spillover effect will cause instability of the closed loop system [19]. In addition, Non-collocation of sensor and actuator is often suitable for installation convenience. In some cases, it is even recommendable for the controllability and observability of high order modes [20]. These cases, however, lead to the problem of non-minimum phase characteristics, which makes the closed loop system only conditionally stable. Meanwhile, time delay will be inevitably caused. Thus, both the control performance and stability of the system are all affected [21]. As a result, although great progress has been achieved in the field of modeling of smart flexible structures, the issue is still far from completely solved.

Various studies have been conducted on the development and implementation of control algorithms for vibration suppression [22]. Classical feedback control strategies receive considerable 
attention as no adaptation time or reference signal is needed. Among those control methods, the poles placement method (PPM) is an effective approach for active vibration suppression despite its simplicity; it offers users the possibility of placing the closed loop poles at some predetermined locations, which is desirable to set natural frequencies and damping ratios to specified values to achieve desired control performance [23]. Sethi and Song [24] applied the PPM for vibration control of the first dominant mode of flexible composite large I-beams, they further presented multi-mode vibration suppression of a cantilever beam using a poles placement controller with an observer [25]. Tehrani et al. [26] proposed a robust poles placement controller for structural vibration control using receptance data. Gordon and Erkorkmaz [27] studied the use of the poles placement technique to achieve active vibration damping and positioning control of ball screw drives. Tehrani et al. [28] developed a multi-input partial poles placement controller for vibration control of a glass-fiber beam using macro fiber composite (MFC) actuators. As the relocation of closed loop poles will alter the system stability characteristics and transient response, locations of the closed loop poles must be carefully determined.

The remainder of this paper is organized as follows. In Section 2, the experimental setup of the smart flexible manipulator system is introduced. The system identification procedure is described, and the identified results are presented in Section 3. Section 4 briefly describes the proposed poles placement method. Simulations are conducted in Section 5. Subsequently, the experimental results and discussion are presented in Section 6. Conclusions are drawn in Section 7.

\section{Experimental Setup}

The experimental setup of the manipulator system is shown in Fig. 1. The flexible manipulator used in this paper is a uniform aluminum cantilever beam and its properties are listed in Table 1. To fulfill the illustration of structural responses in the low frequency scope, instead of traditional PZT sensors, a full bridge strain gauge arrangement with the parameters detailed in Table 2, is attached on both sides of the beam as schematically illustrated in Fig. 2 . The strain gauge is chosen to lie near the root of the beam where the strain energy of the structure is the highest. Two PZT actuators with the properties listed in Table 3, are bonded onto both sides of the beam near the strain gauge. Moreover, a laser displacement sensor of Micro-Epsilon ILD 1402-50 (range: 50mm, resolution: $5 \mu \mathrm{m}$ ) is used to measure the tip displacement of the manipulator. An electromagnetic vibrator is fixed to excite vibration of the beam as an external disturbance. The real-time control system is implemented using a personal computer. A DAQ device of National Instrument PCI-6221(37 pin) is used for data acquisition and control output. The PZT actuator is driven by a power amplifier, which can amplify the input signals from $\pm 5 \mathrm{~V}$ to $\pm 75 \mathrm{~V}$. The strain signal is low-pass filtered and amplified through a strain amplifier, which can amplify the signal to a voltage range of $\pm 10 \mathrm{~V}$. The output signal of the laser displacement sensor is conditioned to a voltage signal of $1 \sim 5 \mathrm{~V}$ by a signal conditioner. In addition, all the control algorithms are developed with the LabVIEW programming software.

Table 1. Properties of the manipulator

\begin{tabular}{cccc}
\hline Symbol & Quantity & Units & Value \\
\hline$l_{b}$ & length & $\mathrm{mm}$ & 1070.2 \\
$w_{b}$ & width & $\mathrm{mm}$ & 61.6 \\
$t_{b}$ & thickness & $\mathrm{mm}$ & 2.1 \\
$E_{b}$ & Modulus of elasticity & $\mathrm{Gpa}$ & 72 \\
$\rho_{b}$ & density & $\mathrm{Kg} / \mathrm{m}^{3}$ & 2700 \\
\hline
\end{tabular}




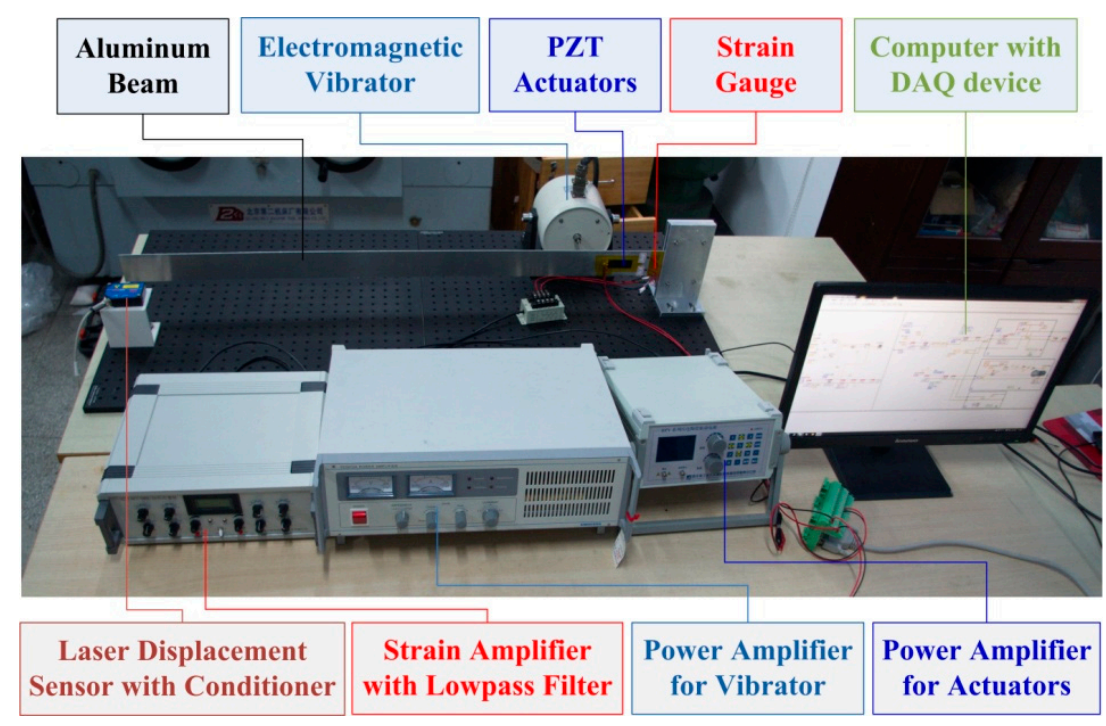

Figure 1. Experimental Setup of the smart manipulator system

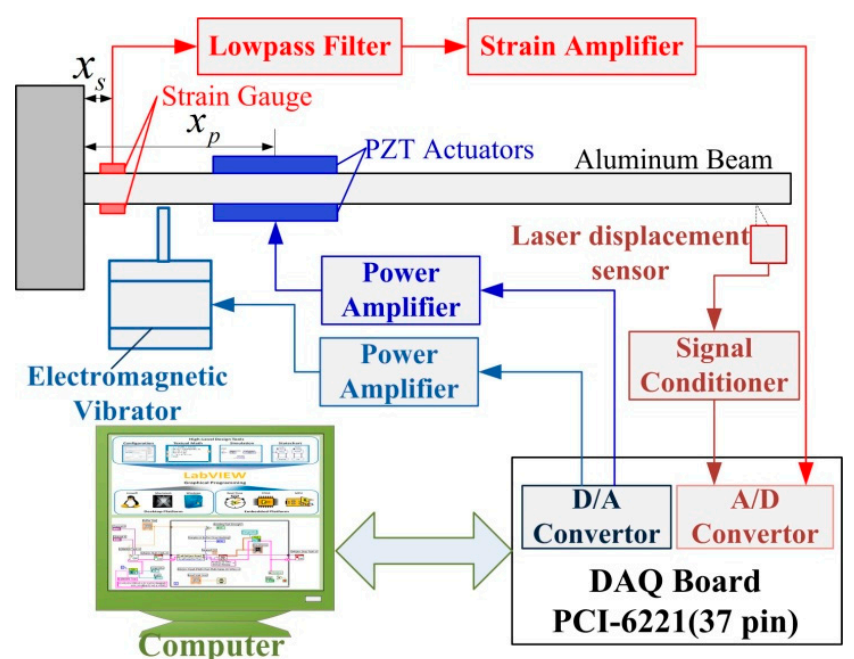

(Processing Unit)

Figure 2. Block diagram of the experimental setup

Table 2. Properties of the strain measurement

\begin{tabular}{cccc}
\hline Symbol & Quantity & Units & Value \\
\hline$S_{g}$ & Gauge sensitivity & -- & 2.08 \\
$R_{s}$ & Resistance & $\Omega$ & 350 \\
$K_{s}$ & Amplification & -- & 5000 \\
$U_{o}$ & Supply Voltage & $\mathrm{V}$ & 4.0 \\
$x_{s}$ & Location on the beam & $\mathrm{mm}$ & 30.0 \\
\hline
\end{tabular}

Table 3. Properties of the PZT patches

\begin{tabular}{cccc}
\hline Symbol & Quantity & Units & Value \\
\hline$l_{p} \times w_{p} \times t_{p}$ & length $\times$ width $\times$ thickness & $\mathrm{mm}$ & $60 \times 20 \times 0.8$ \\
$E_{p}$ & Modulus of elasticity & $\mathrm{Gpa}$ & 63 \\
$\rho_{p}$ & Patch density & $\mathrm{kg} / \mathrm{m}^{3}$ & 7650 \\
$d_{31}$ & Strain coefficient & $\mathrm{C} / \mathrm{N}$ & $-166 \times 10^{-12}$ \\
$x_{p}$ & Location on the beam & $\mathrm{mm}$ & 92.4 \\
\hline
\end{tabular}




\section{System Identification}

System identification can be used to experimentally extract the dynamic model of a system. For system identification, a mathematical model of the beam is necessary. Due to the spatially distributed nature of flexible structures, it is highly desirable to work with transfer functions, thus the vibration dynamics of a cantilever beam can be written as Eq. (1)

$$
G(s)=k \sum_{n=1}^{\infty} \frac{1}{s^{2}+2 \zeta_{n} \omega_{n} s+\omega_{n}^{2}}
$$

where $k$ is the gain of the open loop, $n$ is the mode number, $\zeta_{n}$ and $\omega_{n}$ are the damping ratio and natural frequency of the $n$th mode, respectively. But it is impractical to identify the infinite number of vibration modes. In practice, only a certain bandwidth is of interest, and dynamic response of the flexible structures is often dominated by the vibrations of the lower modes. Therefore, some form of mode truncation for the higher modes is needed. A common reduced order model used by Bhikkaji et al.[15] Orszulik and shan [17] is given as

$$
G_{c}(s)=\sum_{i=1}^{m} \frac{k_{i}}{s^{2}+2 \zeta_{i} \omega_{i} s+\omega_{i}^{2}}+D
$$

where $m$ is the number of modes kept in the model, $k_{i}$ is the gain of the open loop for each mode, $D$ is a feedthrough term, which is added to compensate for the modal truncation effect. But this neglect of higher modes still leads to the appearance of the direct energy transmission [29]. Therefore, the poles of the truncated system, which represent the resonance frequencies, are natural property of the system and independent among different modes, but the locations of the zeros will be distorted due to the omission of the higher modes. Besides, the strain sensors and PZT actuators are not collocated as shown in Fig. 2, these locations may lead to the problem of the non-minimum phase characteristics and some sort of time delay. Above all, the truncated model including the non-collocated sensors/actuators is given by Eq. (3)

$$
G(s)=k e^{-\tau s} \sum_{i=1}^{m} \frac{\left(s-a_{i}\right)\left(s-b_{i}\right)}{s^{2}+2 \zeta_{i} \omega_{i} s+\omega_{i}^{2}}
$$

Where $a_{i}$ and $b_{i}$ are the relocated zeros of the $i$ th mode due to the omission of the higher modes. Meanwhile, a pure del ay element is added to the truncated model representing the time delay, $\tau$ is the delay time.

Using Taylor series expansion, the pure delay element can be approximated as Eq. (4)

$$
e^{-\tau s}=\left[\sum_{j=0}^{\infty} \frac{(\tau s)^{n}}{j !}\right]^{-1}=\left[1+\tau s+\cdots \frac{(\tau s)^{n}}{j !} \cdots\right]^{-1} \approx \frac{1}{1+\tau s}
$$

Then, the truncated model of the smart structure can be rewritten as Eq. (5)

$$
G(s)=\frac{k}{1+\tau s} \sum_{i=1}^{m} \frac{\left(s-a_{i}\right)\left(s-b_{i}\right)}{s^{2}+2 \zeta_{i} \omega_{i} s+\omega_{i}^{2}}
$$

Experiments were conducted to identify the parameters of the system model. A chirp signal with a frequency varies from 0.1 to $15 \mathrm{~Hz}$ was applied to the PZT actuators to have the chance of exciting the first two modes of the structure. The magnitude of the excitation signal was $5 \mathrm{~V}$, and the identification experiment took $15 \mathrm{~s}$. The sampling frequency was $200 \mathrm{~Hz}$, and a low-pass filter was utilized with a cut-off frequency of $100 \mathrm{~Hz}$. The time response signals for chirp excitation are plotted in Figs. 3(a)-(b). 


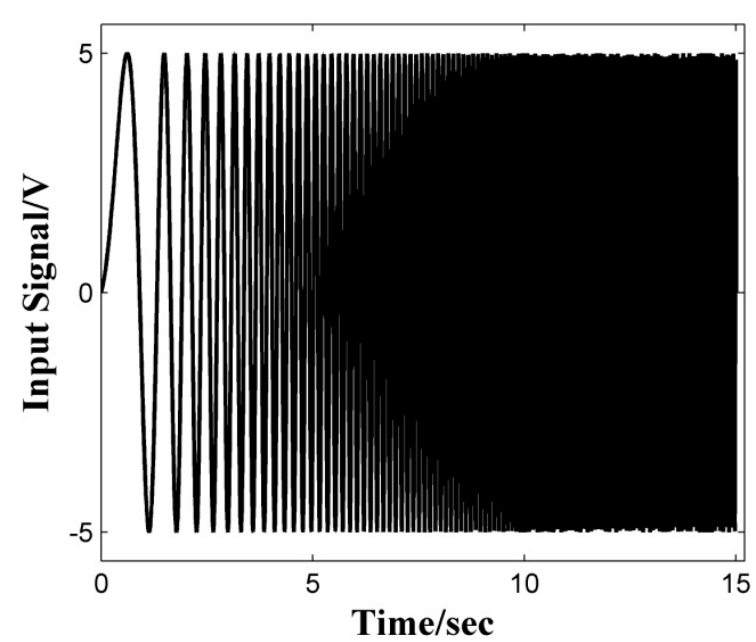

(a) Chirp excitation signal

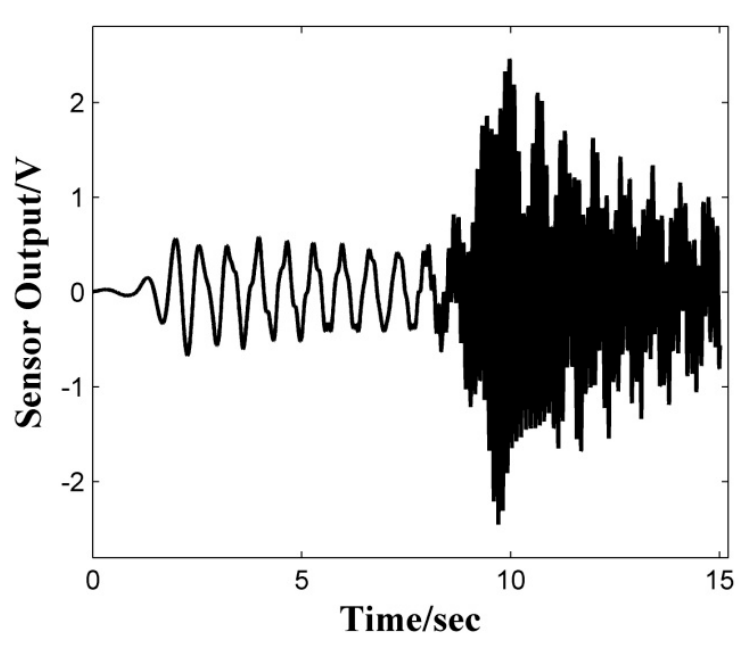

(b) Sensor output signal

Figure 3. Time response of the chirp excitation and measured output signal

To find the transfer function of the system in the frequency domain, Fourier transform is performed on the chirp excitation signal $u(t)$ and sensor output signal $y(t)$, the frequency response function(FRF) of the open loop system is given by Eq. (6)

$$
G(j \omega)=\frac{S_{u y}(j \omega)}{S_{u u}(j \omega)}
$$

Where $S_{u y}(j \omega)$ is the cross spectral density between signal $u(t)$ and $y(t), S_{u u}(j \omega)$ is the auto spectral density of signal $u(t)$. The measured frequency response of the system is plotted in Fig. 4 , it can be seen that the natural frequencies of the first two modes of the smart structure are 1.47 and $9.06 \mathrm{~Hz}$, respectively.

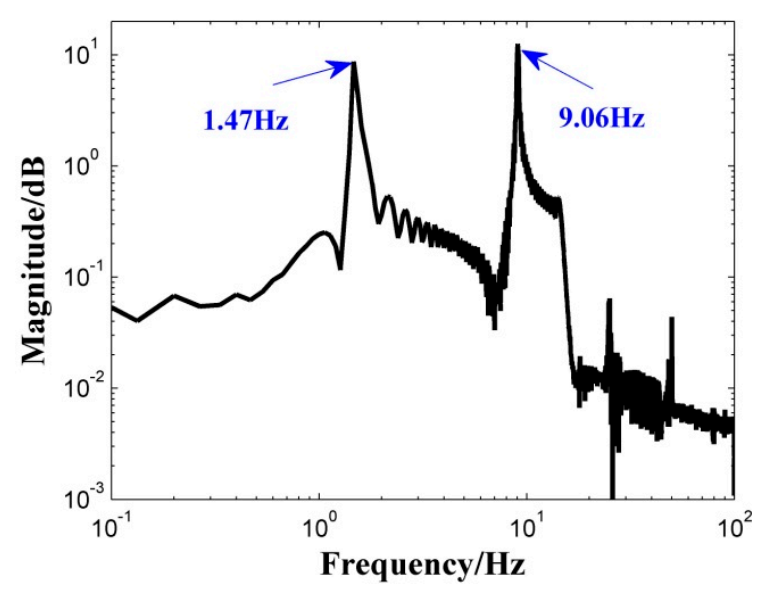

Figure 4. Measured frequency response of the system

The system identification toolbox (Matlab software) was used for system identification. Based on Eq. (5), the identified transfer function using the chirp signal is expressed as Eq. (7). For comparison, the identified transform function depicted as Eq. (2) is also given in Eq. (8). And the modal parameters for the two indentified models are detailed in Table 4. Meanwhile, Bode diagram of the two identified transfer functions in the frequency range of interests $(0.1-100 \mathrm{~Hz})$ are plotted in Fig. 5.

$$
\begin{gathered}
G(s)=\frac{13.44\left(s^{2}+0.72 s+6.66\right)\left(s^{2}+1.58 s+2045\right)}{(s+355.4)\left(s^{2}+0.071 s+86.99\right)\left(s^{2}+0.42 s+3238\right)} \\
G_{c}(s)=\frac{-1.89}{s^{2}+0.071 s+87.05}+\frac{-43.69}{s^{2}+0.40 s+3238}+0.035
\end{gathered}
$$




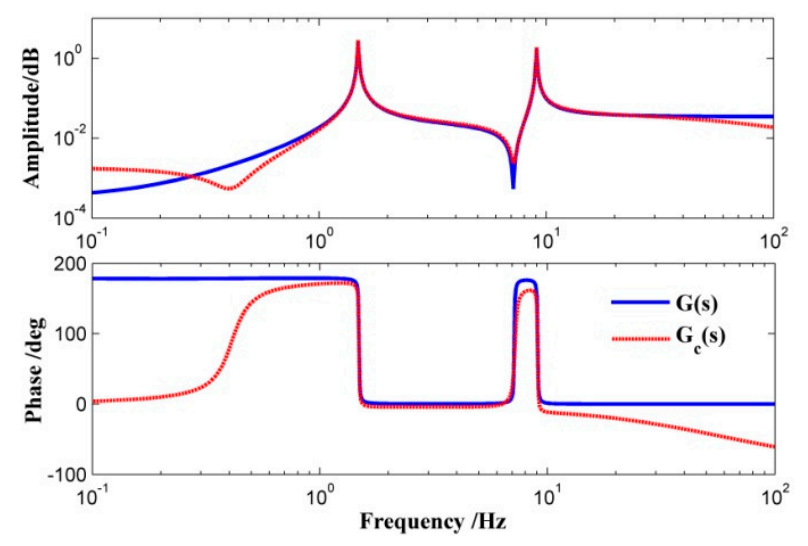

Figure 5. Bode diagrams of the identified models

Table 4. Comparison of modal parameters for the identified models

\begin{tabular}{ccccc}
\multirow{2}{*}{ model } & \multicolumn{2}{c}{ natural frequencies } & \multicolumn{2}{c}{ damping ratio } \\
\cline { 2 - 5 } & $f_{1}$ & $f_{2}$ & $\zeta_{1}$ & $\zeta_{2}$ \\
$G_{c}(s)$ & 1.48 & 9.06 & 0.004 & 0.004 \\
$G(s)$ & 1.48 & 9.06 & 0.004 & 0.004 \\
\hline
\end{tabular}

It can be observed from Table 4 that the first two resonance frequencies of the identified models are both 1.48 and $9.06 \mathrm{~Hz}$ respectively, which are nearly the same as the results obtained from the measured frequency response in Fig. 4. Meanwhile, the two identified models have nearly the same damping ratios for the first two modes. Bode diagrams of the two identified models have similar characteristics nearby the resonance frequencies, as shown in Fig. 5. The locations of the zeros and poles for the two models are also given in Fig. 6. It is worthwhile to note that the locations of the poles of the two identified models coincide highly, while the locations of the zeros of the two identified models are totally different; this is because the locations of the zeros are distorted due to the omission of the higher modes, different system models lead to different relocation of the zeros as depicted before.

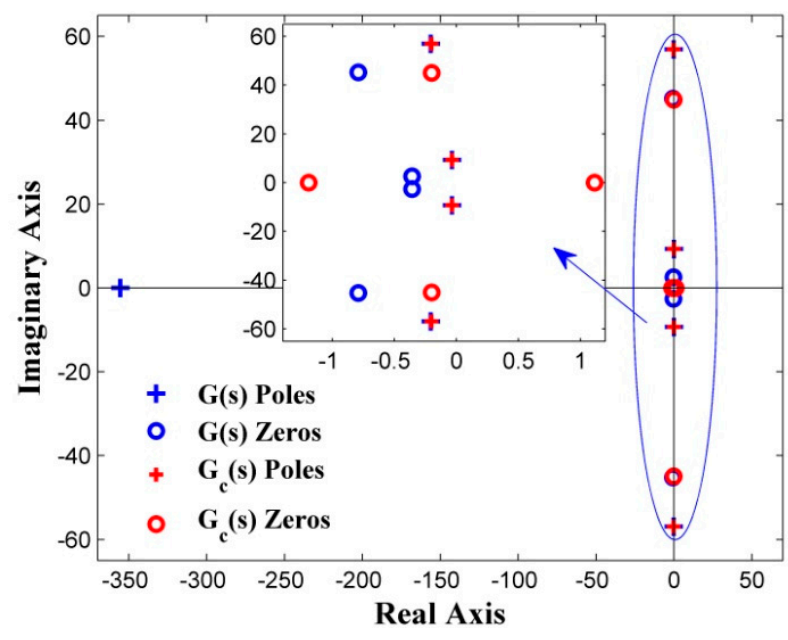

Figure 6. Locations of the zeros and poles

System responses of the two identified models excited by the same chirp signal are illustrated in Figs. 7(a)-(b), both in the time and frequency domains. Besides, the measured system response of the smart structures is also given for comparison. As seen from these figures, system responses of the two identified models are both in good agreement with the experimental response. An excellent match between the identified models and experimental results can be noticed nearby the two resonance frequencies. However, at the beginning period of the chirp excitation $(t<1.5 \mathrm{~s})$, there are 
some differences between the model $G_{c}(s)$ and the measured system responses. It is noticed that system responses of the identified model $G(s)$ show a better agreement with the experimental responses than that of the model $G_{c}(s)$. As a result, the identified model $G(s)$ is accurate both in the time and frequency domains.

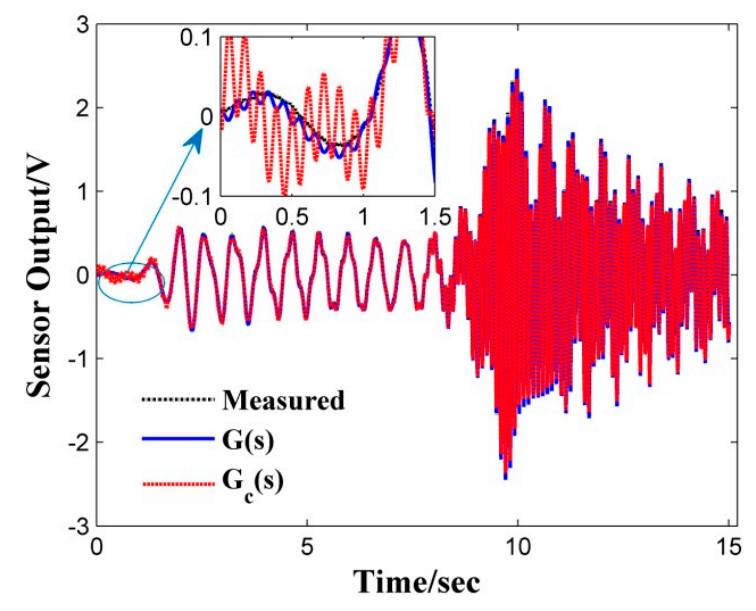

(a) Time responses

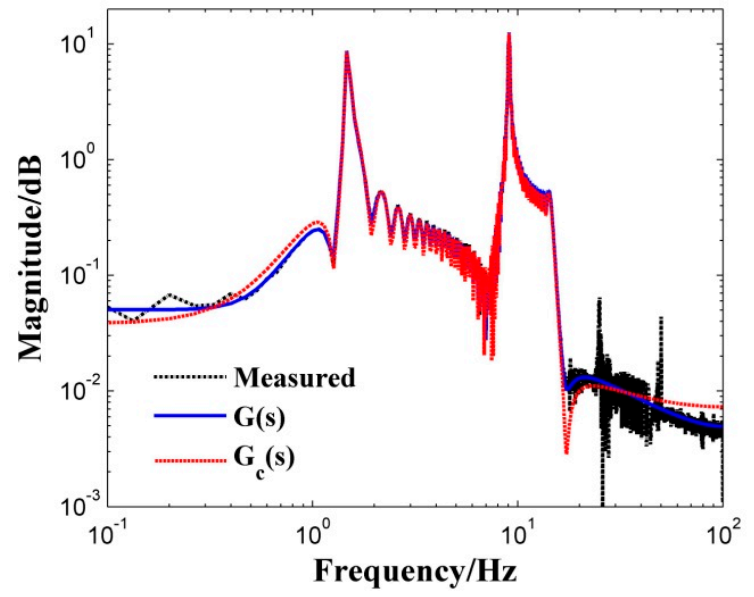

(b) Frequency responses

Figure 7. Comparison of system responses with the chirp signal excitation

The non-minimum phase characteristics of the smart structure are clearly seen in the phase plot of Fig. 5, due to the non-collocation of sensors and actuators. In addition, since the identified delay time $\tau(0.003 \mathrm{~s})$ is far less than $1 \mathrm{~s}$, it is reasonable that the pure delay element is approximated by a first-order inertia element in Eq. (3).

After system model has been identified, it is necessary to validate whether the model is precise enough to represent the real system. The validation experiment is very required since a good match on one signal alone does not necessarily mean it is well matched on another [30]. For this test, the plant was subjected to a summation excitation of several sinusoidal signals of $1.6 \mathrm{~V}$, their frequencies were at $0.5 \mathrm{~Hz}, 1.5 \mathrm{~Hz}, 6 \mathrm{~Hz}$ and $9 \mathrm{~Hz}$, respectively. The time responses for the validation signal are plotted in Figs. 8(a)-(b).

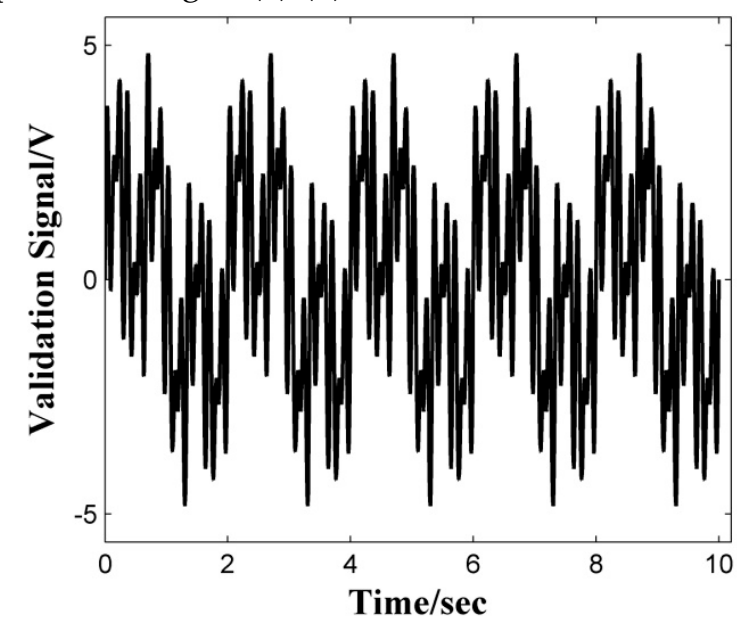

(a) Input signal

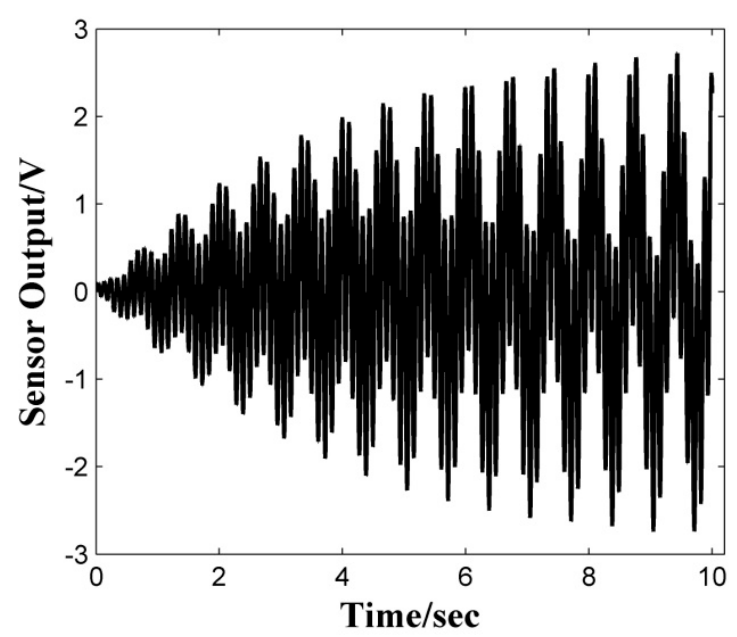

(b) Sensor output signal

Figure 8. Time response of the validation excitation and measured output signal

A comparison between the actual structure and the identified transfer function when subjected to the validation signal is shown in Figs. 9(a)-(b). It can be seen that the output signals are very close, thus confirming that the system identification has performed properly. And the fitness percentage (FP) between the real output and estimated output was calculated for quantitative comparison, which is defined as Eq. (9). 


$$
F P=1-\sqrt{\frac{\sum_{i=1}^{N}\left(\mathrm{y}_{i}-\hat{\mathrm{y}}_{i}\right)^{2}}{\sum_{i=1}^{N} \mathrm{y}_{i}^{2}}}
$$

Where $N$ is the number of the acquisition data, $y_{i}$ is the measured system response, $\hat{y}$ is the estimated response of the identified model. The calculation results are summarized in Table 5.

Table 5. Comparison of FP of identified models

\begin{tabular}{ccc}
\hline Identified model & Chirp excitation & Validation excitation \\
\hline$G_{c}(s)$ & $77.9 \%$ & $81.4 \%$ \\
$G(s)$ & $97.2 \%$ & $90.5 \%$ \\
\hline
\end{tabular}

It is clearly observed from the validation test that the identified model considered in this study performs well. Comparing the FP in Table 5, it is noted that the proposed model G(s) performs better than the widely used model $G_{c}(s)$. Consequently, the identified model $G(s)$ with the delay element is chosen to represent the real plant.

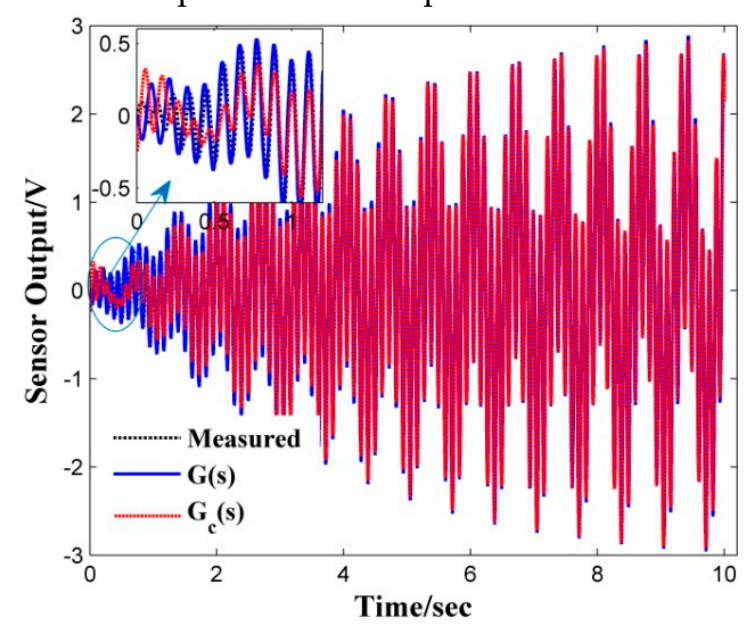

(a) Time responses

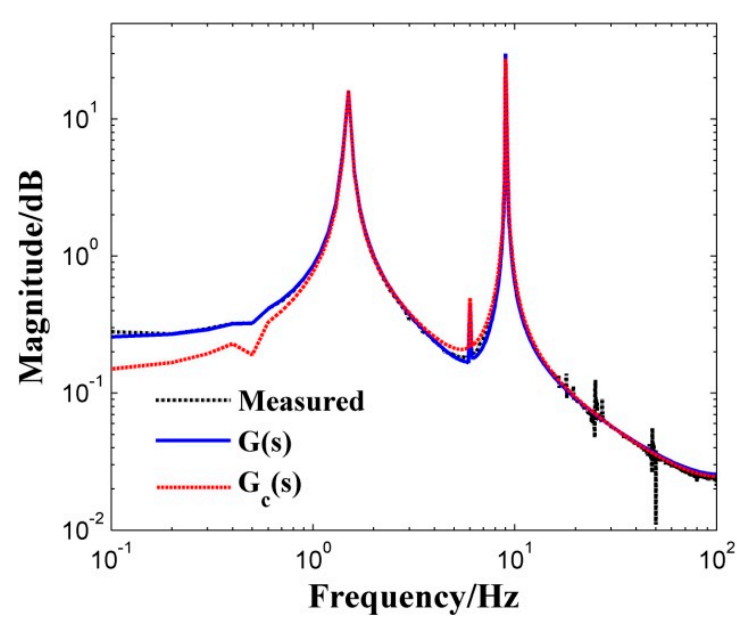

(b) Frequency responses

Figure 9. Comparison of system responses with the validation signal excitation

\section{Controller Design}

The poles placement method (PPM) is a well-known approach employed in the feedback control. It offers the flexibility to relocate the dominate poles of the closed loop system to achieve desired performances. PPM is very desirable for feedback control, as the location of the poles corresponds directly to the eigenvalues of the system, which controls the characteristics of the closed loop response [31]. It is known that the poles of symmetric structural systems are complex with real and complex parts. The poles of the proposed smart structure are related to modal damping ratios and natural frequencies by Eq. (10)

$$
\left\{\begin{array}{l}
\lambda_{1,2}=-\xi_{1} \omega_{1} \pm j \omega_{1} \sqrt{1-\xi_{1}^{2}}=-\xi_{1} \omega_{1} \pm j \omega_{d 1} \\
\lambda_{3,4}=-\xi_{2} \omega_{2} \pm j \omega_{2} \sqrt{1-\xi_{2}^{2}}=-\xi_{2} \omega_{2} \pm j \omega_{d 2}
\end{array}\right.
$$

Except the pole corresponding to the inertia element of the identified model $G(s)$, the locations of the poles corresponding to the first two modes are plotted in Fig. 10. 


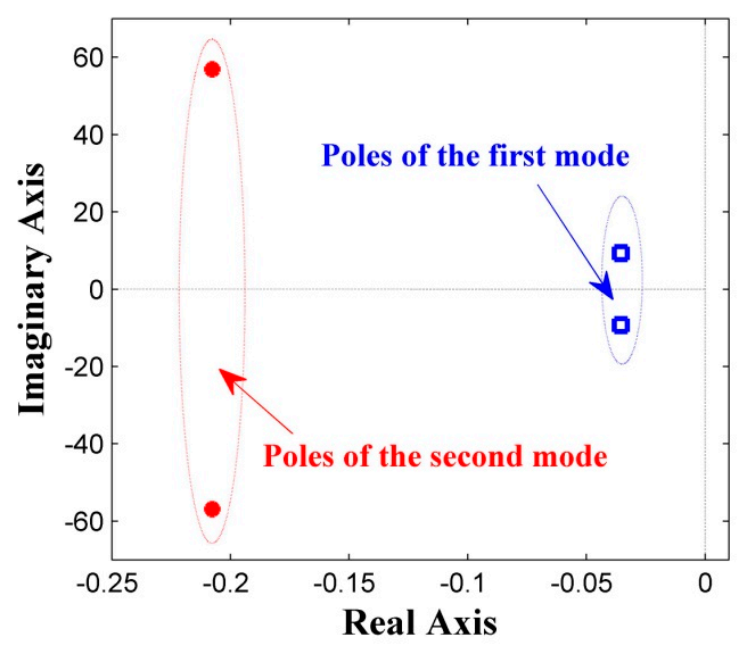

Figure 10. Locations of poles corresponding to the first two modes

Mathematically, if the dominant complex-conjugate poles lie close to the imaginary axis, the system transient response exhibits excessive oscillations and it may be very slow. Therefore, to guarantee fast and well-damped transient response characteristics, it is necessary to place the closedloop poles in a particular region far away from the imaginary axis. It can be observed from Fig. 10 that both the two pairs of poles locate fairly close to the imaginary axis. Thus, both the two pairs of poles need to be relocated to get a better performance of the system [32].

Compared with the polynomial form, the state space approach is more convenient in designing the controller. As it offers the designer to select $\mathrm{n}$ independent gains for a system, the arbitrarily desired poles is achieved. The state space representation of the system is written as Eq. (11)

$$
\begin{aligned}
& \dot{\boldsymbol{x}}(t)=\boldsymbol{A} \boldsymbol{x}(t)+\boldsymbol{B} u(t) \\
& \mathrm{y}(t)=\boldsymbol{C} \boldsymbol{x}(t)+\boldsymbol{D} u(t)
\end{aligned}
$$

Where $\mathrm{y}(t)$ is the output vector, in our case is the voltage output of strain sensors, and $u(t)$ is the input vector, it is the pre-amplified voltage to the piezoelectric actuators. $x(t), A, B, C$ and $D$ are the state variable vector, state matrix, control matrix, output matrix and direct transfer matrix, respectively. Those parameters are defined as

$$
\boldsymbol{x}(t)=\left[\begin{array}{c}
x_{1} \\
\vdots \\
x_{i} \\
\vdots \\
x_{n}
\end{array}\right]=\left[\begin{array}{c}
\dot{x}_{2}+p_{1} y(t)-q_{1} u(t) \\
\vdots \\
\frac{d x_{i+1}(t)}{d t}+p_{i} y(t)-q_{i} u(t) \\
\vdots \\
y(t)
\end{array}\right], \boldsymbol{A}=\left[\begin{array}{ccccc}
0 & 0 & \ldots & 0 & -p_{0} \\
1 & 0 & \ldots & 0 & -p_{1} \\
0 & 1 & \ldots & 0 & -p_{2} \\
\vdots & \vdots & & \vdots & \vdots \\
0 & 0 & \ldots & 1 & -p_{n-1}
\end{array}\right], \boldsymbol{B}=\left[\begin{array}{c}
q_{0} \\
q_{1} \\
q_{2} \\
\vdots \\
q_{n-1}
\end{array}\right], \boldsymbol{C}=\left[\begin{array}{c}
0 \\
0 \\
0 \\
\vdots \\
1
\end{array}\right], \boldsymbol{D}=0
$$

Where $n$ is the degree of the denominator of the identified model $G(s)$, and in our case is 5. $p_{n}, p_{n-}$ $1, \ldots, p_{1}$ and $p_{0}$ are the coefficients of the denominator of $G(s)$ in descending powers of $s . q_{n}, q_{n-1}, \ldots, q_{1}$ and $q_{0}$ are the coefficients of the numerator of $G(s)$.

The state space equations must be controllable in order to implement the PPM. Thus, system observability and controllability matrices are calculated to ensure all the states are controllable and observable. Once those are determined, poles of the closed loop system could be placed at any desired location by means of state feedback through an appropriate state feedback gain matrix. The feedback control $u(t)$ can be defined as Eq. (13)

$$
u(t)=-\boldsymbol{K} \boldsymbol{x}(t)
$$

Where $K$ is the state feedback gain matrix. Substituting Eq. (13) into Eq. (11) gives Eq. (14)

$$
\dot{\boldsymbol{x}}(t)=(\boldsymbol{A}-\boldsymbol{B} \boldsymbol{K}) \boldsymbol{x}(t)
$$


The eigenvalues of matrix $(A-B K)$ are the closed loop poles of the controlled system, which determine the closed loop performance of the system. If the locations of the desired poles are determined, the feedback gain matrix can be solved using Eq. (15)

$$
|s \boldsymbol{I}-(\boldsymbol{A}-\boldsymbol{B} \boldsymbol{K})|=\prod_{i=1}^{g}\left(s-p_{i}\right)
$$

Where $p_{i}$ is the desired closed loop poles, $g$ is the poles number.

For effective vibration suppression, it is necessary to choose the closed loop poles appropriately. In practice, if the desired closed loop poles are placed far from the left of the imaginary axis, the voltage delivered by the controller might exceed the actuator limits, thus possibly destabilizes the closed loop system; Furthermore, the generated voltage may oscillate too fast in order to control the system, and a noise amplification will be caused, which leads to a possible excitation of the high frequency modes of the system. Consequently, the spillover effect is unavoidable. On the other hand, a close location of closed loop poles may lead to a slow closed loop response. Therefore, a compromise needs to be found between the response time and control force.

To achieve the desired damping characteristics of the target modes, without affecting the natural frequencies of the structure, the best solution is to adjust only the real part of the dominant poles. As it is known, the dynamic response of a second order system has no vibration and achieves a stable state in a short time when the damping ratio is 0.707 . So the optimal locations of the desired poles can be attained when the closed loop damping ratio is set to be 0.707 . The basic idea of this method is illustrated in Fig. 11. The comparisons between the relocated closed loop and open loop poles are listed in Table 6.

Table 6. Comparisons between the closed loop and open loop poles

\begin{tabular}{cccc}
\hline Open loop poles & Open loop damping ratio & Closed loop poles & Close loop damping ratio \\
\hline$-0.036 \pm 9.33 \mathrm{i}$ & 0.004 & $-6.59 \pm 9.33 \mathrm{i}$ & 0.707 \\
$-0.21 \pm 56.9 \mathrm{i}$ & 0.004 & $-40.23 \pm 56.9 \mathrm{i}$ & 0.707 \\
\hline
\end{tabular}

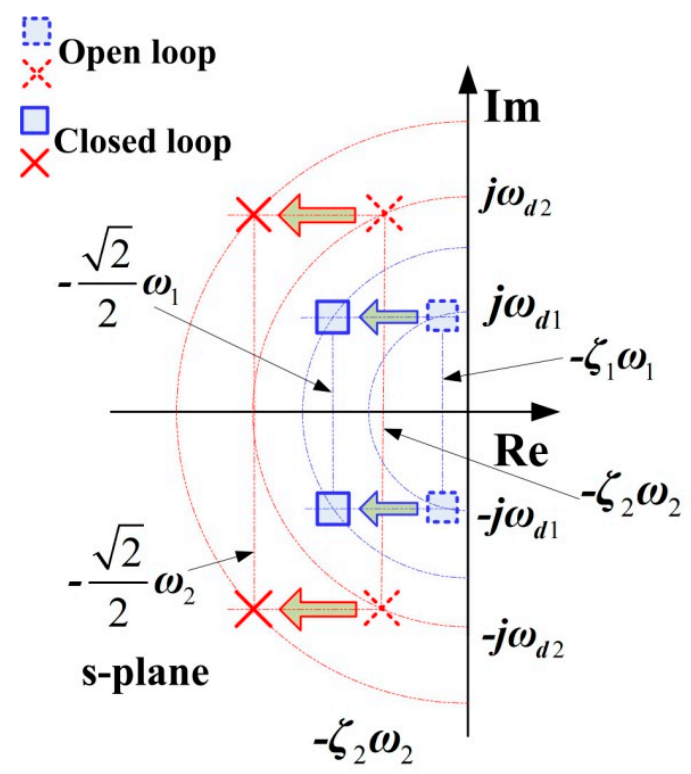

Figure 11. Optimal locations of the closed loop poles

\section{Simulations}

To evaluate the effectiveness of the proposed controller, simulations of system response in the closed loop were performed with the above derived transfer function $G(s)$. The excitation signal was a summation of the two sinusoidal signals of modal frequencies for a period of $10 \mathrm{~s}$ at $0.5 \mathrm{~V}$. A saturation block with limits of $\pm 5 \mathrm{~V}$ was incorporated to limit the control effort, as it would be in 
implementation. The simulated time responses for the open and closed loop system in a total period of $40 \mathrm{~s}$ are shown in Fig. 12.

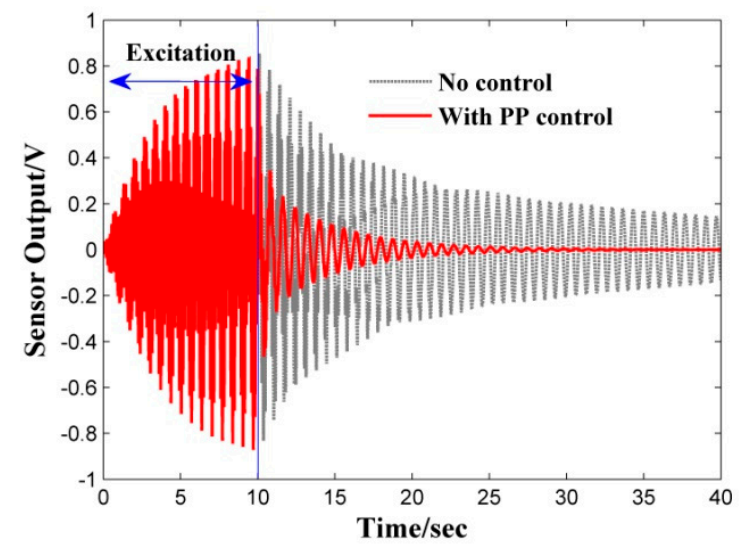

Figure 12. Simulated time responses for open and closed loop system

As plotted in Fig. 12, the excited vibrations for the open loop system take more than $30 \mathrm{~s}$ to eliminate, while with the poles placement controller, the system vibrations die down in less than 15 s. obviously, system vibrations are effectively suppressed. Furthermore, two sets of optional locations for the closed loop poles as given in Table 7, are chosen to verify the effectiveness of the proposed controller.

Table 7. Comparisons of optional locations for closed loop poles

\begin{tabular}{ccc}
\hline Optional location & Closed-loop poles & damping ratio \\
\hline Closer location & $-3.73 \pm 9.33 \mathrm{i}$ & 0.4 \\
& $-22.76 \pm 56.9 \mathrm{i}$ & \\
Optimal location & $-6.59 \pm 9.33 \mathrm{i}$ & 0.707 \\
& $-40.23 \pm 56.9 \mathrm{i}$ & \\
Further location & $-7.93 \pm 9.33 \mathrm{i}$ & 0.85 \\
\hline
\end{tabular}

After the locations of the desired poles are determined, Eq. (15) can be rewritten as

$$
|s \boldsymbol{I}-(\boldsymbol{A}-\boldsymbol{B} \boldsymbol{K})|=\left(s-p_{d}\right) \prod_{i=1}^{2}\left(s-p_{i}\right)\left(s-\hat{p}_{i}\right)
$$

Where $p_{i}$ and $\hat{p}_{i}$ are the complex-conjugate poles, and $p_{d}$ is the pole corresponding to the first-order inertia element. Then, the feedback gain matrix can be solved using Eq. (16).

Simulation results with different locations of closed loop poles are illustrated in Figs. 13(a)-(b). It can be seen that a further location of the closed loop poles leads to a better control performance, but a severer spillover phenomenon is caused. On the other hand, the spillover phenomenon is avoided with a closer location of the closed loop poles, but the effectiveness of the controller decreases unavoidably. 


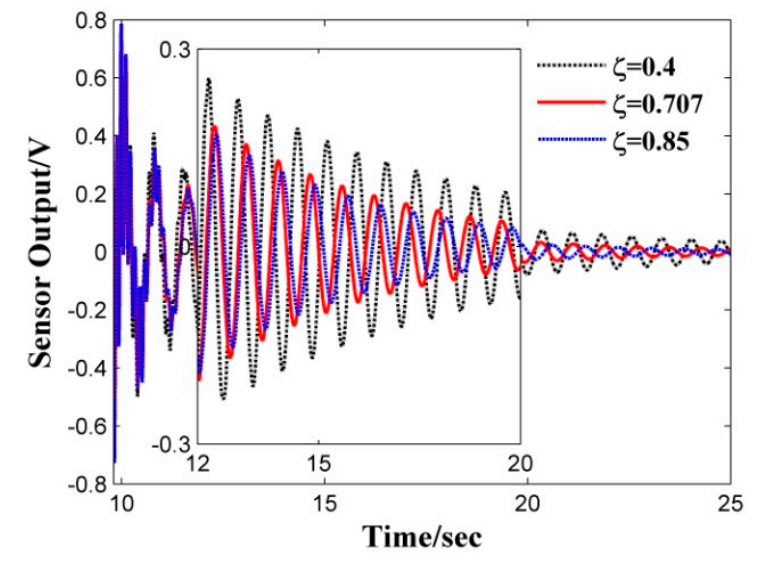

(a) Time response of the closed loop system

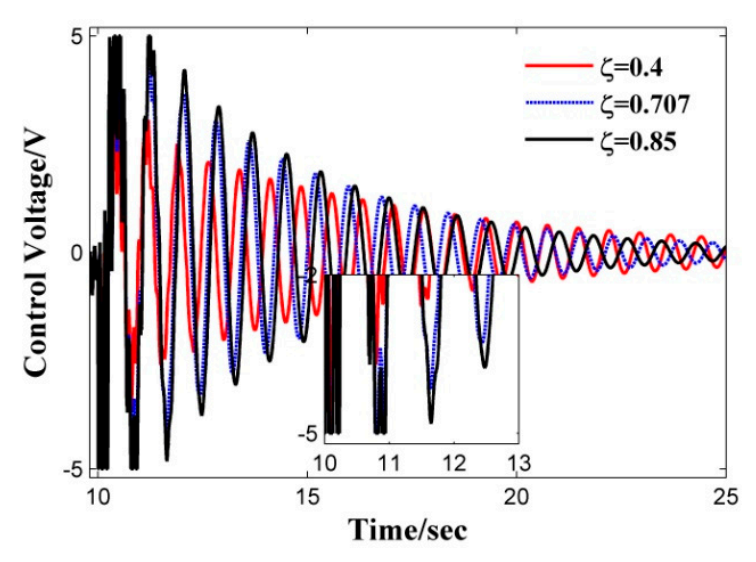

(b) Control voltage of PZT actuators

Figure 13. Simulation results with different locations of the closed loop poles

\section{Experiments}

The identified model combined with the proposed controller was implemented on the experimental setup as. The controller was tested in the real time data acquisition system with the same sampling frequency of $200 \mathrm{~Hz}$. The experimental setup was excited using the same excitation signal used in simulation. The open loop test was conducted to view the results in the absence of the controller. And the comparisons of the free vibrations (without control) results between simulation and experiment are shown in Fig. 14. It is clear that the experimental results are in good agreement with simulation for the open loop test. It should be pointed out that the amplitude of the time response in simulation is a little larger than that in experiment. This is because the identified model $G(s)$ corrects for the neglected modes.

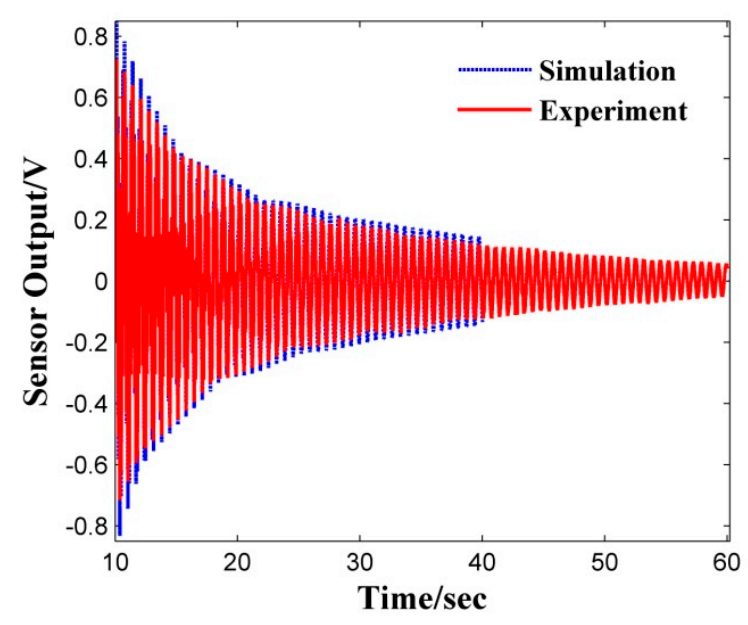

Figure 14. comparisons of free vibration results between simulation and experiment

In addition, the tip displacement measured by the laser displacement sensor is plotted in Fig. 15. It can be seen that the uncontrolled tip displacement of the manipulator still has an amplitude of $0.25 \mathrm{~mm}$, after a damping period of $60 \mathrm{~s}$. The time taken for the vibration to settle down is so long that vibration control must be carried out to accelerate this process. 


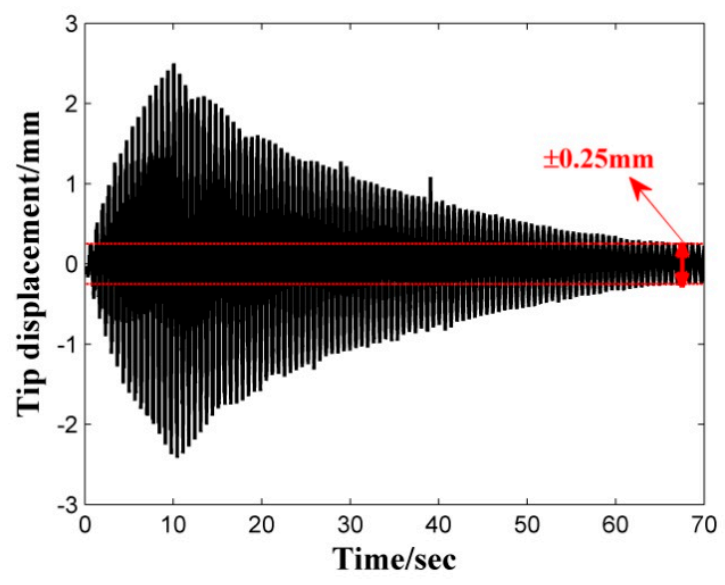

Figure 15. Tip displacement measured by the laser displacement sensor

Next, the controller with the optimal locations of desired poles was implemented. The closed loop time responses with the PPM are shown in Fig. 16(a). It can be seen that the system vibrations are well suppressed with the proposed controller, and the damping period of the vibration response is shortened significantly. Furthermore, the power spectral density comparisons for $10 \mathrm{~s}$ to $25 \mathrm{~s}$ are shown in Fig. 16(b). Compared with the responses of the open loop, the measured magnitude at the first resonant frequency drops from $5.6 \mathrm{~dB}$ to $4.1 \mathrm{~dB}$, and a drop of $2.3 \mathrm{~dB}$ is observed for the second resonant frequency. Evidently, substantial drops are observed at the first two resonant frequencies. Moreover, the closed loop time responses for the simulated and experimental results mach closely as shown in Fig. 17. It is noted that the control efficiency in experiment is a little better than that in simulation. This is because the amplitude of time response in simulation is a little larger than that in experiment as shown in Fig. 14. And the measured tip displacement is shown in Fig. 18. After a damping period of $13 \mathrm{~s}$, the amplitude of the controlled tip displacement reduces to $0.05 \mathrm{~mm}$. Thus, a quick vibration convergence is achieved with the proposed controller.

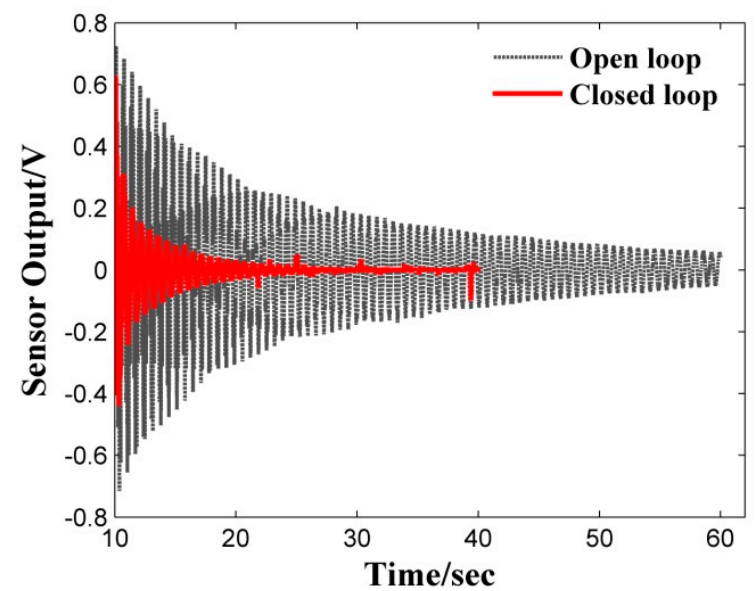

(a) Time response

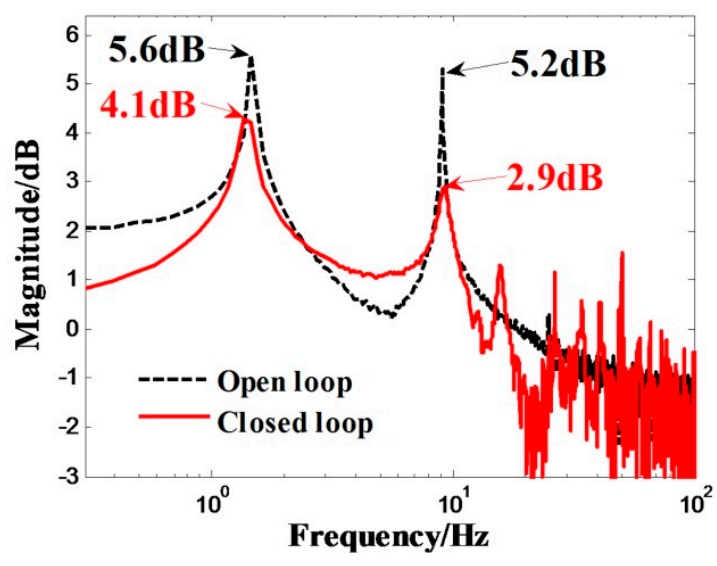

(b) power spectral density

Figure 16. Comparisons of experimental responses between the open and closed loop 


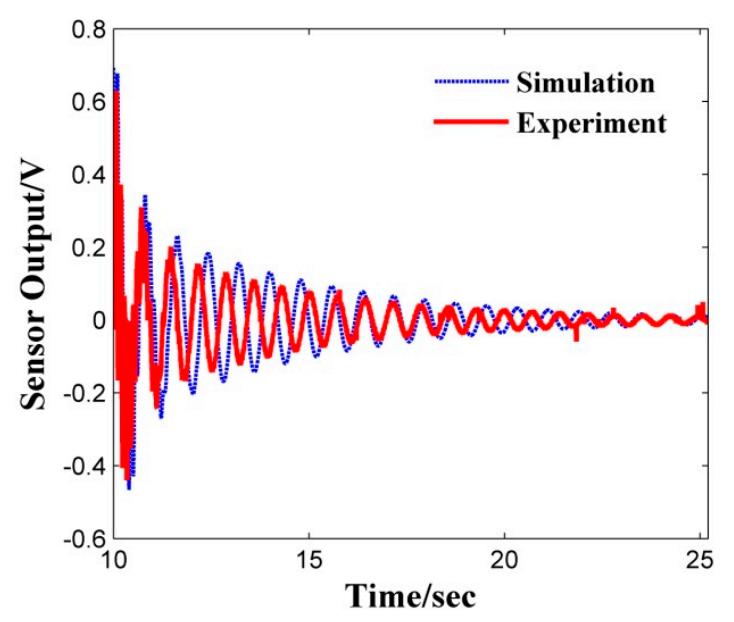

Figure 17. Comparisons of the closed loop time responses

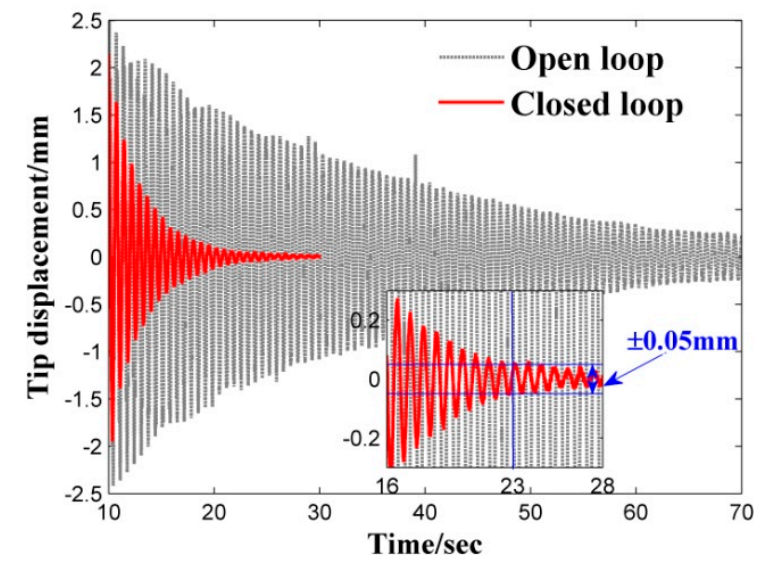

Figure 18. Comparisons of the measured tip displacement

To further evaluate the performance of the controller, the same structure was subjected to an external disturbance by the electromagnetic vibrator (EMV). The open loop (without control) and closed loop (with the controller) time responses are shown in Fig. 19(a). In the absence of the controller, the uncontrolled response measured by the strain sensors still has an amplitude of $0.14 \mathrm{~V}$ after a damping period of $50 \mathrm{~s}$, while now is suppressed to $0.05 \mathrm{~V}$ within $9 \mathrm{~s}$. and the power spectral density comparisons for a period of $25 \mathrm{~s}$ are presented in Fig. 19(b).

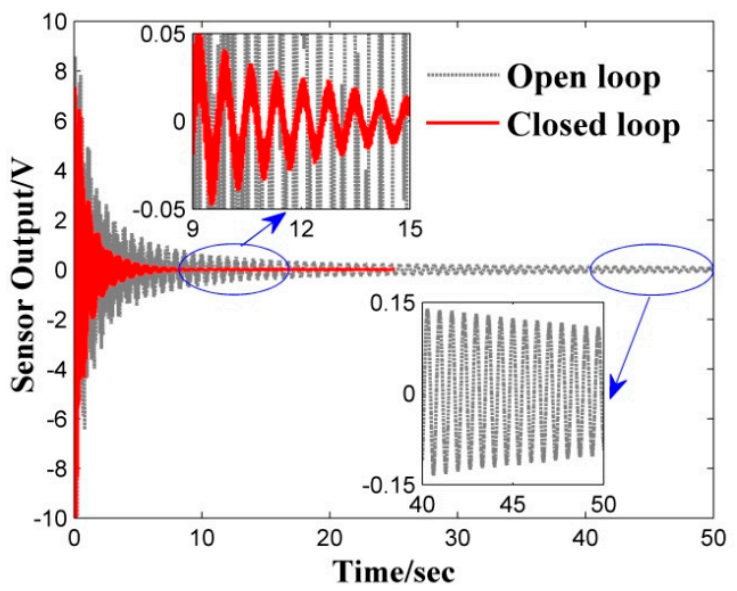

(a) Time response

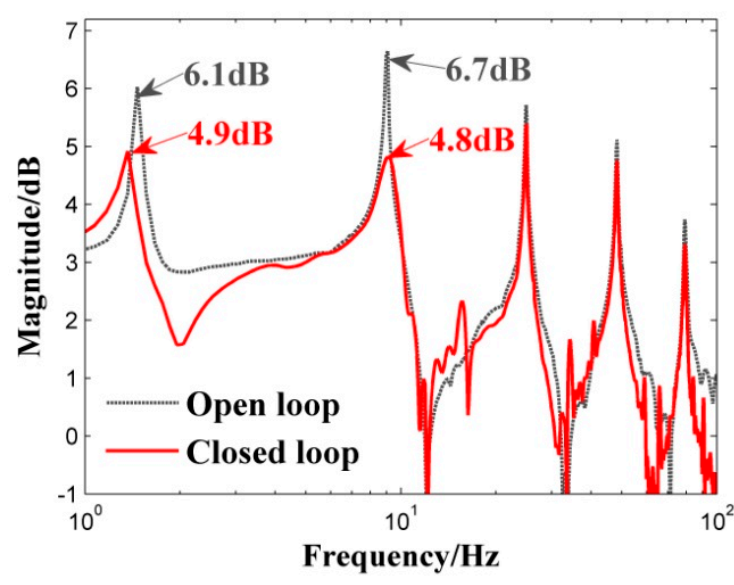

(b) power spectral density

Figure 19. Comparisons of experimental responses excited by the EMV

It is noticed that the first two vibration modes are well suppressed. This is expected to happen since the first two modes, corresponding to the first two pairs of dominant poles, are the ones that are mostly needed to be diminished. Moreover, it is also interesting to notice from Fig. 19(b) that the 
three higher modes (the third, fourth and fifth peaks), excited by the impulse excitation of the EMV, also experience a small attenuation. Even though the corresponding poles have not been changed, this might be the influence of the first two pairs of poles that have been relocated. As the three higher vibration modes have fast convergence speed in the time domain, they are not dominant to affect the system response. The induced vibrations die down very quickly with the proposed controller as compared in Fig. 20.

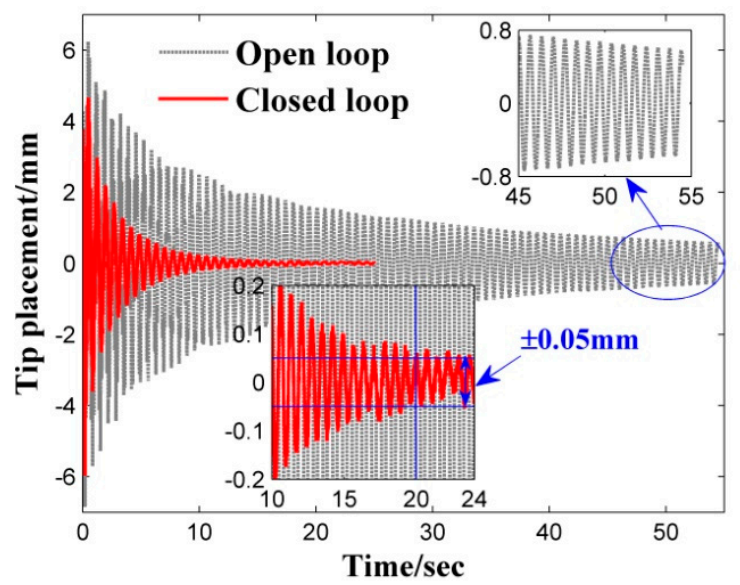

Figure 20. Comparisons of measured tip displacement excited by the EMV

The amplitude of the controlled tip displacement reduces to $0.05 \mathrm{~mm}$ after a damping period of $13 \mathrm{~s}$, while the damping period takes more than $60 \mathrm{~s}$ without the controller. Besides, it is necessary to notice as the peak value of the measured vibration response exceeds the range of the A/D board $( \pm 10$ V) as plotted in Fig. 19(a); control saturation of actuators is inevitable. The control voltage applied on actuators is depicted in Fig. 21.

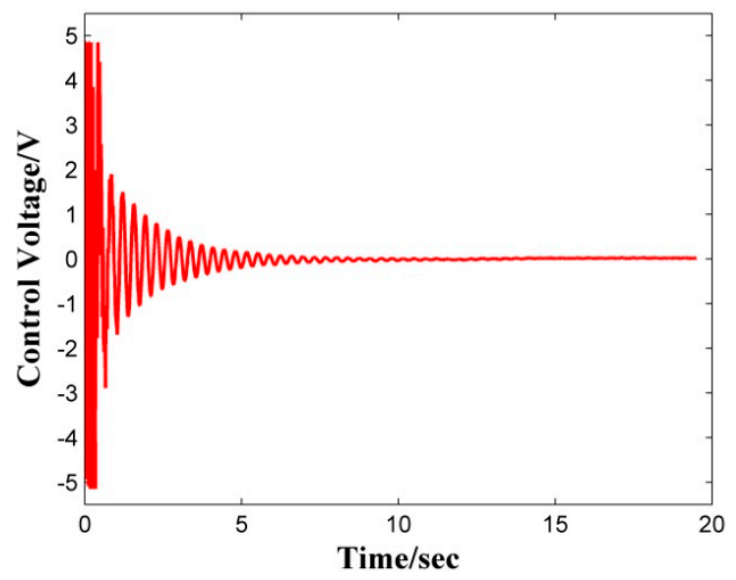

Figure 21. Control voltage applied on actuators

Finally, experimental results of the poles placement controller with different locations of the closed loop poles are compared in Figs. 22(a)-(d). Significant attenuation of measured time responses measured by the strain sensors can be seen for the two cases, namely the further location of poles with damping ratio $0.85(-48.37 \pm 56.9 \mathrm{i},-7.93 \pm 9.33 \mathrm{i})$ and the optimal location of poles with damping ratio $0.707(-40.23 \pm 56.9 \mathrm{i},-6.59 \pm 9.33 \mathrm{i})$. 


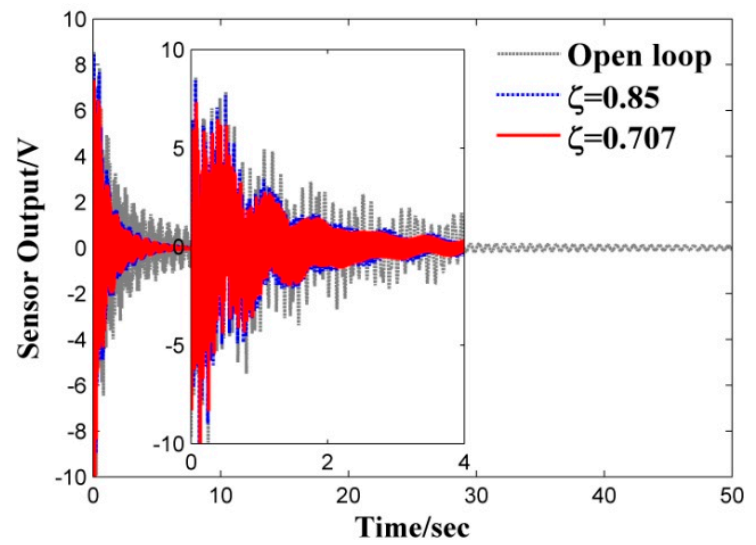

(a) Measured time response by strain sensors

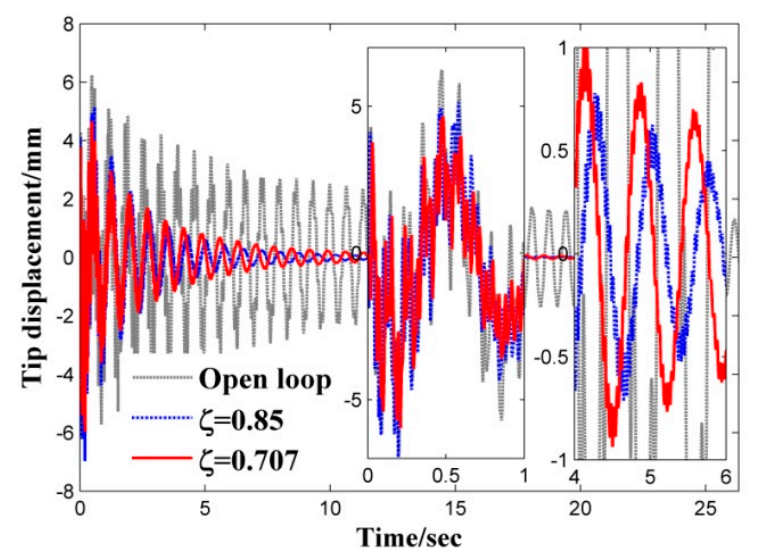

(c) Measured tip displacement

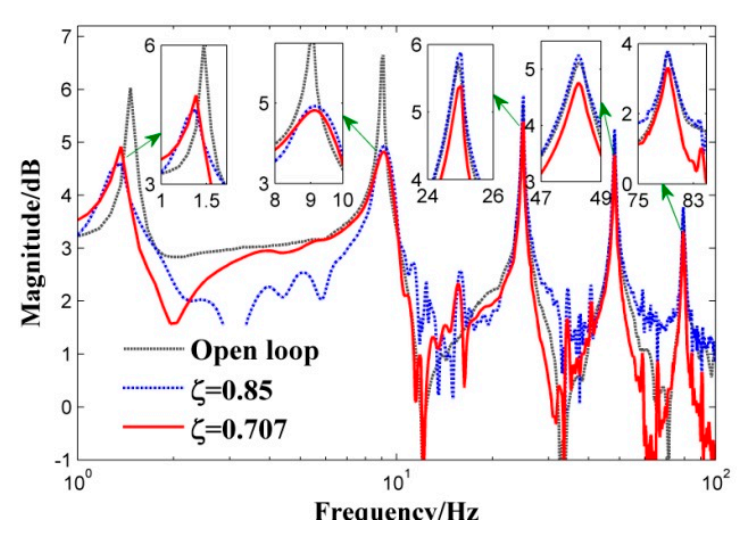

(b) power spectral density

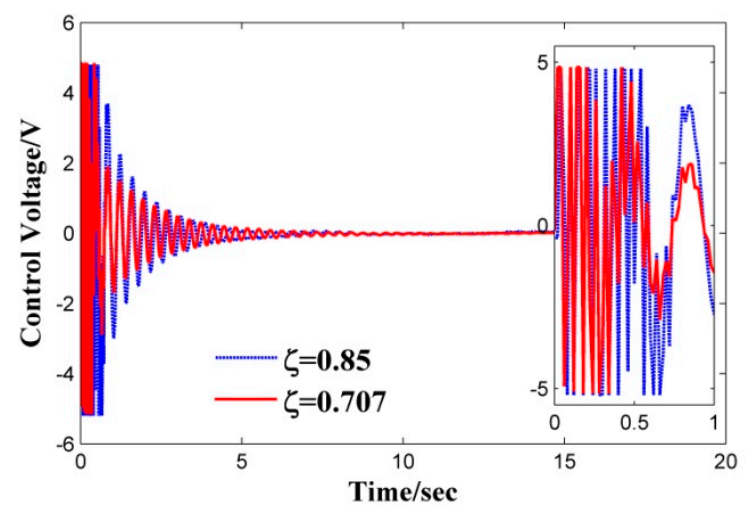

(d) Control voltage applied on the actuators

Figure 22. Comparisons of experimental results with different locations of poles

However, it is important to point out that the measured time responses with the further location of poles decays a little slower than that with the optimal location of poles in Fig. 22(a). The experimental results show the control effectiveness reduces with a further location of poles, which is different from simulations. In the case of further location of poles, it is observed that vibration responses of the first two modes are well suppressed, and a larger attenuation of the first mode is achieved in Fig. 22(b), compared with the optimal case. But peaks corresponding to the other three higher modes (the third, fourth and fifth peaks) have larger amplitudes than that in the open loop case. All these can be attributed to the fact that the desired closed loop poles are placed too far from the imaginary axis; hence the control voltage delivered by the controller severely exceeds the system limits, which lead to a severe control saturation phenomenon as demonstrated in Fig. 22(d). Furthermore, the spillover effect is caused by the control saturation, and vibrations of the higher modes are excited. Those results are further illustrated by the measured vibration response of the beam tip in Fig. 22(c). At the beginning period (0-1s), vibrations of the higher modes are excited due to the severe spillover effect, thus the peak values of tip displacement with the further location of poles are slightly larger than that with the optimal case. As vibrations of the higher modes converge quickly in the time domain, the spillover effect disappears during the periods of 4-6s, and the control force of the actuators is mainly used to deal with vibrations of the first two modes. Correspondingly, the control effectiveness with the further location of poles is better than that with the optimal case in the period of $4-6 \mathrm{~s}$.

In summary, locations of the closed loop poles must be carefully determined to implement the poles placement controller. The effectiveness of the optimal multi-poles placement method is validated by experiments. Multi-mode vibrations of the smart flexible manipulator are well suppressed with the proposed controller. 


\section{Conclusions}

Experimental identification and multi-mode vibration suppression of a smart flexible manipulator bonded with non-collocated piezoelectric actuators and strain sensors are conducted in this paper. A reduced order transfer function representing the physical system is proposed, and a first-order inertia element is added to the model for experimental identification. An optimal multipoles placement controller is proposed and implemented on the structure. Both comparative simulated and experimental results validate the effectiveness and feasibility of the proposed controller. The conclusions of this study can be summarized as follows:

In order to descirbe the smart flexible manipulator system, a reduced order transfer function model with relocated zeros compensating for the neglect of higher modes is proposed. And a firstorder inertia element representing the non-collocation of the sensors and actuators is added to the model for system identification. Comparative results show the identified model match closely with the experimental results both in the time and frequency domains, and a fit of $97.2 \%$ is achieved in the identification results. The validation test with an excitation of several sinusoidal signals further validated the excellent performance of the proposed model.

An optimal multi-poles placement controller is designed and implemented on the structure. The optimal locations of the closed loop poles are determined at the best damping ratio 0.707 . Compared with different locations of poles, the best performance of the optimal locations of poles is demonstrated by simulations. Experimental results show vibrations of the first two modes of the manipulator are significantly diminished as expected. Moreover, vibrations of the higher modes are also slightly suppressed with the proposed controller. In addition, if a further location of poles is chosen, the spillover effect will be caused due to the control saturation of actuators, and vibrations of the higher modes will be excited. Consequently, the best performance for the optimal locations of the closed loop poles is validated, and the effectiveness and feasibility of the proposed controller are demonstrated.

Acknowledgments: This work was supported by the National Natural Science Foundation of China (No. 51505238\&51375433) and Zhejiang Provincial Natural Science Foundation of China (No. LQ15E050002). The authors would also like to thank the support of K.C. Wong Magna Fund in Ningbo University.

Conflicts of Interest: The authors declare no conflict of interest.

\section{References}

1. Zhao Z L, Qiu Z C and Zhang X M. Vibration control of a pneumatic driven piezoelectric flexible manipulator using self-organizing map based multiple models. Mech Syst Signal Pr. 2016, 70-71 345-372

2. $\quad \mathrm{Xu} Z$, Shan X, Chen D, et al.. A Novel Tunable Multi-Frequency Hybrid Vibration Energy Harvester Using Piezoelectric and Electromagnetic Conversion Mechanisms. Appl. Sci. 2016, 6(1) 10

3. Lou J Q, Wei Y D, Yang Y L and Xie F R. Hybrid PD and effective multi-mode positive position feedback control for slewing and vibration suppression of a smart flexible manipulator. Smart Mater Struct. 2015, 24035007

4. Sethi V, Franchek M A and Song G B. Active multimodal vibration suppression of a flexible structure with piezoceramic sensor and actuator by using loop shaping. J Vib Control. 2011, 17 1994-2006

5. Jonathan B, Ramón T J, Vicente F and Hebertt S R. Adaptive Controller for Single-Link Flexible Manipulators Based on Algebraic Identification and Generalized Proportional Integral Control. IEEE T Syst Man Cy B. 2009, 39 735-751

6. Tornabene F, Fantuzzi N, Bacciocchi M, et al. An Equivalent Layer-Wise Approach for the Free Vibration Analysis of Thick and Thin Laminated and Sandwich Shells. Appl. Sci. 2016, 7(1) 17

7. Saad M S, Jamaluddin H and Darus I Z M. Active vibration control of a flexible beam using system identification and controller tuning by evolutionary algorithm. J Vib Control 2013, 21 2027-2042

8. Bu X Z, Ye L, Su Z Q and Wang C H. Active control of a flexible smart beam using a system identification technique based on ARMAX. Smart Mater Struct 2003, 12 845-850

9. Abreu G L C M, Conceicao S M, Brennan M J and Alves M T S. System Identification and Active Vibration Control of a Flexible Structure. J Braz Soc Mech Sci Eng. 2012, 34 386-392 
10. Tavakolpour A R, Darus I Z M, Tokhi O and Mailah M. Genetic algorithm-based identification of transfer function parameters for a rectangular flexible plate system. Eng Appl Artif Intel. 2010, 23 13881397

11. Afshari S S, Nobahari H and Kordkheili S A H. Experimental Parametric Identification of a Flexible Beam Using Piezoelectric Sensors and Actuators. Shock Vib. 2014, 2014718140

12. Pradhan S K and Subudhi B. Nonlinear Adaptive Model Predictive Controller for a Flexible Manipulator: An Experimental Study. IEEE T Contr Syst T. 2014, 22 1754-1768

13. San-Millan A and Feliu V. A Fast Online Estimator of the Two Main Vibration Modes of Flexible Structures from Biased and Noisy Measurements. IEEE-ASME T Mech. 2015, 20 93-104

14. Qiu Z C, Wu H X and Ye C D. Acceleration sensors based modal identification and active vibration control of flexible smart cantilever plate. Aerosp Sci Technol. 2009, 13 277-290

15. Bhikkaji B, Moheimani S O R and Petersen I R. A Negative Imaginary Approach to Modeling and Control of a Collocated Structure. IEEE-ASME T Mech. 2012, 17 1-11

16. Ahmadizadeh S, Montazeri A and Poshtan J. Design of minimax-linear quadratic Gaussian controller using the frequency domain subspace identified model of flexible plate. J Vib Control. 2015, 21 11151143

17. Orszulik R R and Shan J J. Experimental study on active vibration control using genetic algorithmbased system identification and optimized positive position feedback. Smart Mater Struct. 2012, 21 125014

18. Bandopadhya D and Njuguna J. A Study on the Effects of Kalman Filter on Performance of IPMCBased Active Vibration Control Scheme. IEEE T Contr Syst T. 2010, 18 1315-1324

19. Kumar R. Efficient Active Vibration Control of Smart Structures with Modified Positive Position Feedback Control Using Pattern Search Methods in the Presence of Instrumentation Phase Lead and Lag. J Dyn Syst-T ASME. 2013, 135 1012-1030

20. Kim S M and Oh J E. A modal filter approach to non-collocated vibration control of structures. J Sound Vib 2013, 332 2207-2221

21. Chen L X, Li S H, Liu K, Cai G P and Li H G. Time delay control of hysteretic composite plate Smart Mater Struct. 2015, 24045046

22. Demetriou D, Nikitas N. A Novel Hybrid Semi-Active Mass Damper Configuration for Structural Applications. Appl. Sci. 2016, 6(12) 397.

23. Abdelaziz T H S. Parametric eigenstructure assignment using state-derivative feedback for linear systems. J Vib Control. 2012, 18 1809-1827

24. Sethi V and Song G B. Pole-Placement Vibration Control of a Flexible Composite I-beam using Piezoceramic Sensors and Actuators. J Thermoplast Compos. 2006, 19 293-307

25. Sethi V and Song G B. Multimodal Vibration Control of a Flexible Structure using Piezoceramic Sensor and Actuator. J Intel Mat Syst Str. 2008, 19 573-582

26. Tehrani M G, Mottershead J E, Shenton A T and Ram Y M. Robust pole placement in structures by the method of receptances. Mech Syst Signal Pr. 2011, 25 112-122

27. Gordon D J and Erkorkmaz K. Accurate control of ball screw drives using pole-placement vibration damping and a novel trajectory prefilter. Precis Eng. 2013, 37 308-322

28. Tehrani M G, Elliott R N R and Mottershead J E. Partial pole placement in structures by the method of receptances: Theory and experiments. J Sound Vib. 2010, 329 5017-5035

29. Orszulik R R and Shan J J. Active vibration control using genetic algorithm-based system identification and positive position feedback. Smart Mater Struct. 2012, 21055002

30. Tavakolpour A R, Darus I Z M, Tokhi O and Mailah M. Genetic algorithm-based identification of transfer function parameters for a rectangular flexible plate system. Eng Appl Artif Intel. 2010, 23 13881397

31. Mottershead J E, Tehrani G M, James S and Court P. Active vibration control experiments on an AgustaWestland W30 helicopter airframe. P I Mech Eng C-J Mec. 2012, 226 1504-1516

32. John E. Mottersheada J E, Tehrania M G, Jamesa S and Ramb Y M. Active vibration suppression by pole-zero placement using measured receptances. J Sound Vib. 2008, 311 1391-1408 